\title{
(SUSY) Higgs Search at the LHC
}

\author{
M. Margarete Mühlleitner \\ LAPTH, 9 Chemin de Bellevue, B.P.110, 74941 Annecy-Le-Vieux, Cedex, France
}

\begin{abstract}
The discovery of the Standard Model (SM) or supersymmetric (SUSY) Higgs bosons belongs to the main endeavors of the Large Hadron Collider (LHC). In this article the status of the signal and background calculations for Higgs boson production at the LHC is reviewed.
\end{abstract}

Keywords: Higgs Bosons, Supersymmetry, LHC PACS: $14.80 . \mathrm{Bn}, 14.80 . \mathrm{Cp}$

\section{INTRODUCTION}

The Higgs mechanism is a cornerstone of the SM and its SUSY extensions [1] and renders the search for Higgs boson(s) a crucial endeavor at the LHC. In the SM one Higgs doublet has to be introduced to realize electroweak symmetry breaking (EWSB), leading to one elementary Higgs boson [2]. Experiment and theory constrain the Higgs boson mass range to $114.4 \mathrm{GeV}$ up to $\sim 1 \mathrm{TeV}$ [3]. The minimal supersymmetric extension of the SM (MSSM) requires at least two complex Higgs doublets, implying 5 physical Higgs states after EWSB, two neutral CP-even, $h, H$, one neutral CPodd $A$ and two charged Higgs bosons $H^{ \pm}$. Negative direct searches at LEP2 impose lower mass bounds of $m_{h, H} \gtrsim 92.8 \mathrm{GeV}, m_{A} \gtrsim 93.4 \mathrm{GeV}$ and $m_{H^{ \pm}} \gtrsim 78.6 \mathrm{GeV}$ [4]. SUSY implies an upper light neutral Higgs mass bound of $m_{h} \lesssim 135 \mathrm{GeV}$ [5], including the one-loop and dominant two-loop corrections. The heavier Higgs boson masses range up to $\mathscr{O}(1 \mathrm{TeV})$. At tree level, the MSSM Higgs sector can be parametrized by two independent input parameters, $m_{A}$ and $\operatorname{tg} \beta=v_{2} / v_{1}$, the ratio of the two vacuum expectation values.

\section{GLUON FUSION}

The gluon fusion processes

$$
g g \rightarrow \Phi \quad\left(\Phi=H^{\mathbf{S M}}, h, H, A\right)
$$

are the dominant production mechanisms for the SM Higgs boson in the entire mass range up to $\sim 1 \mathrm{TeV}$ and for MSSM Higgs states for small to moderate values of $\operatorname{tg} \beta$ (see Fig.11). It is mediated by heavy quark triangle loops, and in addition by squark loops in SUSY theories, if $m_{\tilde{q}} \lesssim 400 \mathrm{GeV}$. The next-to-leading order (NLO) QCD corrections, including the full quark mass dependence, increase the production cross section by up to $100 \%$ [6]. The calculation in the heavy top quark limit
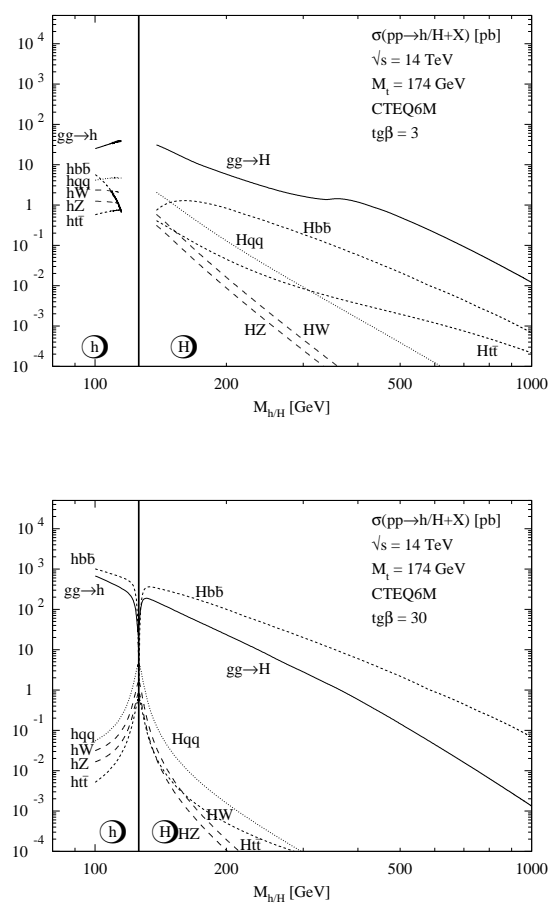

FIGURE 1. Neutral CP-even MSSM Higgs production cross sections at the LHC for gluon fusion $(g g \rightarrow h / H)$, vector boson fusion $(q q h / H)$, Higgs-strahlung $(h V / H V)$, and the associated production $(b \bar{b} h / H, t \bar{t} h / H)$, including QCD corrections except for $b \bar{b} h / H$. Upper: $\operatorname{tg} \beta=3$, lower: $\operatorname{tg} \beta=30$. From Ref. [1].

[7] has been shown to provide an approximation within $10 \%$ in the SM and $20-30 \%$ in the MSSM for $\operatorname{tg} \beta \lesssim 5$ [8]. For large $\operatorname{tg} \beta$ the bottom loop contributions become dominant due to the strongly enhanced bottom Yukawa couplings, so that the large loop mass limit is not applicable any more. In this case, the NLO corrections are of more moderate size, $\mathscr{O}(10-60 \%)$. The next-to-next-to leading order (NNLO) corrections, provided in the heavy top limit, increase the cross section moderately by another 20-30\% [9]. The effect of the dominant finite top 
mass corrections on the approximate NNLO result has been investigated recently in Ref. [10]. Soft gluon resummation leads to a further increase of about $6 \%$ [11] and the estimate of the next-to-next-to-next-to leading order (NNNLO) effects indicates improved perturbative convergence [12]. Two-loop electroweak (EW) corrections add $\sim 5-8 \%[13]$. As for the MSSM processes, the NLO QCD corrections to squark loops have been first known in the heavy squark limit [14], and the full SUSY-QCD corrections in the heavy SUSY particle mass limit [15]. They are large for the squark loops, whereas the genuine SUSY-QCD corrections, mediated by virtual gluino and stop exchanges, are small, of $\mathscr{O}(5 \%)$. The calculation of the QCD corrections to squark loops including the full mass dependence has been performed recently indicating that the squark mass effects on the $K$-factor, describing the ratio between the NLO and LO cross section, can be up to $\sim 20 \%$ with a remaining residual theoretical uncertainty of less than about 20\% [16], see Fig. 2] (The virtual corrections have been derived in Ref. [17].) Recently, the NLO SUSY-QCD corrections including the full SUSY particle mass dependences have been calculated [18].

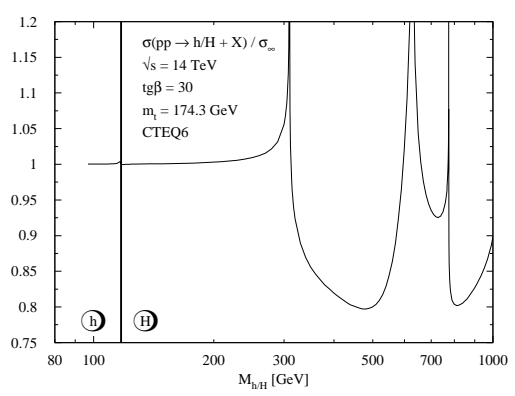

FIGURE 2. Ratio of the QCD corrected scalar MSSM Higgs production cross sections via gluon fusion including the full squark mass dependence and those obtained by taking the relative QCD corrections to the squark loops in the heavy mass limit as functions of the corresponding Higgs masses for $\operatorname{tg} \beta=30$. The kinks and spikes correspond to the various squark pair thresholds. From Ref. [16].

The impact of the higher order (HO) calculations on the rate and the shape of the corresponding distributions may strongly depend on the choice of cuts. This has therefore to be studied to perform reliable experimental analyses. The transverse momentum $p_{T}$ distribution of the Higgs boson plays an important role for the experimental search strategies. At LO Higgs bosons are produced with vanishing transverse momentum in the gluon fusion process. They acquire non-zero $p_{T}$ only if an additional gluon is radiated. This contribution is part of the real NLO corrections to the total gluon fusion cross section. The $p_{T}$ distributions are known up to NLO in the heavy quark limit [19], so that they are valid only for small and moderate Higgs masses and $p_{T}$. For $p_{T}$ values much smaller than $M_{H}$ large logarithms appear which ne- cessitate resummation in order to get a reliable description of the small $p_{T}$ range. This has been done at different levels of theoretical accuracy [20]. Finally, the NLO corrections with two tagged jets at large $p_{T}$ are known in the large $m_{t}$ limit [21] and have been implemented in parton level Monte Carlo programs.

For the MSSM, the Higgs plus 1 jet process is known at LO only [22, 23]. The SM results at NLO QCD for the $p_{T}$ distributions and the resummation of the soft gluon effects can only be applied for small values of $\operatorname{tg} \beta, M_{H}$ and $p_{T}$. For large $\operatorname{tg} \beta$ values, the bottom loops are dominant and as an important consequence the $p_{T}$ distributions of the neutral Higgs bosons are softer than for small values of $\operatorname{tg} \beta$ [23].

As for the NNLO accuracy the inclusive SM cross section with a jet veto was provided some time ago [24]. In the meantime the NNLO result fully taking into account experimental cuts has been performed in the $H \rightarrow \gamma \gamma$ [25] and in the $H \rightarrow W^{+} W^{-} \rightarrow l^{+} l^{-} v \bar{v}$ [26] decay mode. An independent calculation for these and the $H \rightarrow Z Z \rightarrow 4$ leptons decay channel has been finished recently [27] and implemented in a Monte Carlo program.

Search Channels \& Backgrounds: Higgs particles with masses below $\sim 140 \mathrm{GeV}$ have to be searched for in their $\gamma \gamma$ decay channel, the $b \bar{b}$ final state being overwhelmed by the QCD background. The $\gamma \gamma$ irreducible background has been calculated up to NLO including fragmentation effects [28]. The $g g \rightarrow \gamma \gamma$ contribution, which is enhanced by the large gluon luminosity, is known up to NLO [29].

For SM Higgs masses $140 \lesssim M_{H} \lesssim 180 \mathrm{GeV}$ the $H \rightarrow W^{+} W^{-} \rightarrow l^{+} l^{-} v \bar{v}$ decay mode is among the most promising for an early discovery. Challenging due to the large background, the strong angular correlations between the charged leptons from the $W$ decay [30] can be exploited to suppress the background. Since the Higgs mass cannot be reconstructed directly due to the escaping neutrinos a background extrapolation from sidebands is impossible. It has to be extrapolated from the signalfree region and therefore requires a precise knowledge of the background distributions. The NLO $W^{+} W^{-}$irreducible background is known [31] including spin correlations, and the effects of multiple soft-gluon emissions up to next-to-leading-log (NLL) accuracy [32]. The knowledge of spin correlations is crucial for a correct prediction of angular distributions, and they are implemented in the MC@NLO event generator [33]. Also the potentially large gluon initiated contribution $g g \rightarrow W^{+} W^{-}$is available at LO [34]. Recently the NLO QCD corrections to $W^{+} W^{-} j$ production, an important background also to the $W W$ fusion Higgs boson production process (see below), has been reported [35]. The important $t \bar{t}$ background is known at NLO [36], including the effect of spin correlations [37]. The inclusion of width effects is 
available at LO only [38].

The gold-plated channel $H \rightarrow Z Z \rightarrow 4$ leptons becomes dominant for SM Higgs bosons with $M_{H} \gtrsim 180 \mathrm{GeV}$. Since the invariant mass of the leptons can be reconstructed, it is much easier to observe, and the background is measurable from the data. To study the Higgs boson properties, an accurate prediction is necessary, however. The irreducible $Z Z$ background has been provided up to NLO including spin correlations [31], the impact of soft gluon effects on signal and background has been examined [39], and the gluon-initiated contribution $g g \rightarrow Z Z$ is available at LO [40]. The full QCD+EW corrections to the $H \rightarrow W^{+} W^{-}(Z Z) \rightarrow 4$ leptons decay have been computed by Ref. [41].

\section{VECTOR BOSON FUSION}

The $W$ and $Z$ boson fusion processes [42]

$$
p p \rightarrow q q \rightarrow q q+W W / Z Z \rightarrow q q H
$$

for SM Higgs boson production are about one order of magnitude smaller than the gluon fusion process and become competitive with the latter for large Higgs masses. The typical signature of a vector boson fusion (VBF) event is given by two hard jets with a large rapidity interval between them. Since the exchanged boson is colourless, there is no hadronic activity between them, and the NLO QCD corrections can be derived from the NLO corrections to deep inelastic lepton-nucleon scattering. They are of $\mathscr{O}(10 \%)$, neglecting small interference effects [43]. Recently, the full EW and QCD corrections have been computed with a typical size of $5-10 \%$. They induce distortions of the distributions at the $10 \%$ level [44].

In the MSSM, the VBF processes play an important role for the light scalar Higgs $h$ close to its upper mass bound, where it becomes SM-like, and for the heavy scalar Higgs $H$ at its lower mass bound. In the other regions the cross sections are suppressed due to additional SUSY-factors in the Higgs couplings. For the pseudoscalar $A$ the process is absent, since it does not couple to gauge bosons at tree level. The NLO QCD corrections to the total cross sections and distributions can be taken from the SM case and are of the same size. The SUSY-QCD [45] and SUSY EW and QCD corrections [46] turned out to be small.

Search Channels \& Backgrounds: The Higgs +2 jets signature, also produced in gluon fusion, is part of the inclusive Higgs signal, but represents a background to the isolation of the Higgs gauge couplings through VBF. The gluon contribution is known at $\mathrm{LO}$ with full top mass dependence [47], and has been recently complemented by the NLO QCD corrections in the heavy top mass limit
[21]. Parton shower effects on the relevant distributions have been evaluated as well [48] and indicate that the discriminating power of the LO results is not significantly changed. Furthermore, the dominant NLO QCD corrections to Higgs plus 3 jet production in vector boson fusion have been presented in [49]. The corrections to the total cross sections are modest, while the shapes of some kinematical distributions change appreciably.

The Higgs decays into $\tau$ lepton pairs constitute an important MSSM Higgs boson discovery channel [50]. Since in VBF Higgs bosons are produced at large transverse momentum, the $\tau^{+} \tau^{-}$invariant mass can be reconstructed with an accuracy of a few $\mathrm{GeV}$, and the background can in principle be measured from the data using a sideband analysis. The most important backgrounds are QCD $Z j j$ and EW $Z j j$ production from VBF. They are known at NLO accuracy [51].

The $H \rightarrow W^{+} W^{-} \rightarrow l^{+} l^{-} v \bar{v}$ decay, important for SM Higgs particle searches in the intermediate mass range [52], is most challenging, because it does not allow a direct Higgs mass reconstruction. The irreducible $W^{+} W^{-}$ background is calculated up to NLO, the corrections are modest [53]. The other important background $t \bar{t}+$ jet is known at NLO [54]. The essential inclusion of the top decay with full spin correlation is missing so far, as well as the possibly relevant finite width effects.

\section{ASSOCIATED PRODUCTION WITH TOP QUARKS}

Associated Higgs production with a top quark pair [55]

$$
p p \rightarrow q \bar{q} / g g \rightarrow \Phi t \bar{t}
$$

plays a significant role for SM Higgs masses below $\sim 150 \mathrm{GeV}$ and in the MSSM only for the light scalar Higgs state $h$. The NLO QCD corrections have been determined [56] and lead to a moderate increase of the total cross section by $\sim 20 \%$ at the LHC, both for the SM and the MSSM case. The relevant parts of final state particle distribution shapes are only moderately affected, i.e. $\mathscr{O}(10 \%)$. The SUSY-QCD corrections to $t \bar{t} h$ production have been calculated in [57, 58] and are of moderate size.

Search Channels \& Backgrounds: While the $t \bar{t} H$ production with subsequent $H \rightarrow b \bar{b}$ decay was previously considered a discovery channel, recent analyses with a more careful background evaluation are not as optimistic and demand for improved signal and background studies based on NLO calculations to reliably analyse the potential of this channel. After the NLO signal [56] and $t \bar{t} j$ [54] predictions, recently the first step towards the full NLO QCD $p p \rightarrow t \bar{t} b \bar{b}+X$ calculation has been accomplished by evaluating the contribution from quark- 
antiquark annihilation [59]. The $t \bar{t} H \rightarrow t \bar{t} \gamma \gamma$ channel on the other hand provides an important contribution to Higgs discovery in the $\gamma \gamma$ final state [60]. It develops a narrow resonance in the invariant $\gamma \gamma$ mass distribution, so that the background can be measured directly from the sidebands.

\section{ASSOCIATED PRODUCTION WITH BOTTOM QUARKS}

While unimportant in the SM, in the MSSM for large values of $\operatorname{tg} \beta$ the Higgs boson production in association with bottom quarks

$$
p p \rightarrow q \bar{q} / g g \rightarrow \Phi b \bar{b} \quad \Phi=h, H, A
$$

constitutes the dominant Higgs production process. Two different formalisms, which represent different orderings of perturbation theory, can be applied to calculate the cross section. In the four-flavour scheme, the LO process is given by $g g \rightarrow b \bar{b} \Phi$. The NLO QCD corrections can be deduced from the analogous calculation with a top quark final state pair and turn out to be large [61]. This may be due to the large logarithms generated by the integration over the transverse momenta of the final state bottom quarks. The NLO corrections with one or two tagged high- $p_{T} b$-jets have been provided as well [61]. To resum the logarithms bottom quark densities have to be introduced in the proton. In the five flavour scheme the LO process is then given by $b \bar{b} \rightarrow \Phi$. The NLO [62] and NNLO [63] QCD corrections to the inclusive process are

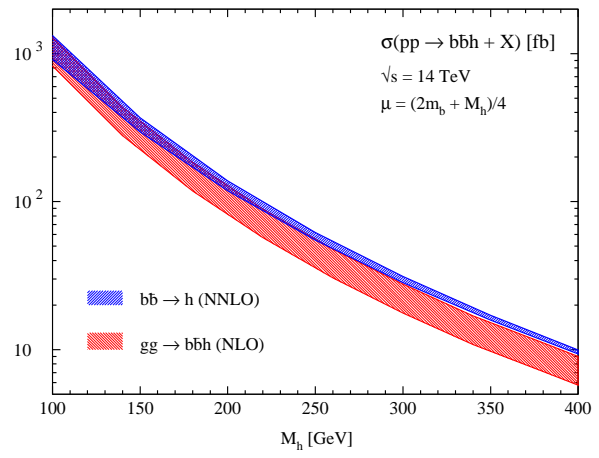

FIGURE 3. Total cross sections $p p \rightarrow b \bar{b} h+X$ as a function of the Higgs mass $M_{h}$ with no $b$-jet identified in the final state. The error bands correspond to varying the renormalization and factorization scale within $\left(2 m_{b}+M_{h}\right) / 8$ and $\left(2 m_{b}+M_{h}\right) / 2$. From Ref.[65].

known and of moderate size. For the process with one tagged high- $p_{T} b$-jet they have been calculated at NLO [64]. At sufficiently high order in perturbation theory, the results of the two approaches should converge against the same value, see Fig. 3 and Ref. [65] for details.
Furthermore, the EW corrections to $b \bar{b} \rightarrow \Phi[66]$ and the Yukawa coupling induced contributions to $g g \rightarrow b \bar{b} h$ at one loop [67] have been calculated. The SUSY-QCD corrections to $b \bar{b} h$ have been given in [58] and turn out to be significant. (Approximative results have been given in Ref. [68].) The SUSY QCD corrections to $\Phi b$ production [69] are typically of order of a few percent after appropriate redefinition of the Yukawa couplings.

\section{HIGGS-STRAHLUNG OFF VECTOR BOSONS}

For SM Higgs masses in the intermediate range $M_{H} \lesssim 2 M_{Z}$ the Higgs-strahlung off $W, Z$ bosons [70]

$$
p p \rightarrow q \bar{q} \rightarrow Z^{*} / W^{*} \rightarrow H+Z / W
$$

provides alternative Higgs boson search signatures. For the neutral MSSM Higgs bosons, however, this process plays no major role at the LHC. The NLO QCD corrections can be inferred from the Drell-Yan process and increase the total cross section by $\mathscr{O}(30 \%)$ [71]. The NNLO corrections [72] lead to an increase of less than $10 \%$ for the relevant Higgs boson masses at the LHC. The full EW corrections decrease the total cross section by 5-10\% [73]. The total theoretical uncertainty is of $\mathscr{O}(5 \%)$. The NLO EW and NNLO QCD corrections are similar in the MSSM case. The SUSY-QCD corrections are small [45], whereas the SUSY-EW corrections are unknown.

\section{CHARGED HIGGS BOSON PRODUCTION}

The dominant charged Higgs boson production process is the associated production with heavy quarks [74]

$$
p p \rightarrow q \bar{q}, g g \rightarrow H^{-}+t \bar{b} \quad \text { and } \quad c . c .
$$

The NLO QCD and SUSY-QCD corrections are significant [75], partly due to logarithms which arise from the integration over the transverse momentum of the final state bottom quark, and due to the large SUSY-QCD corrections to the bottom Yukawa coupling. In analogy to the neutral Higgs boson case, the logarithms can be resummed by introducing bottom quark densities. In this 5-flavour approach the LO process is then given by $g b \rightarrow$ $H^{-} t$ and its charge conjugate. The NLO SUSY-QCD corrections to this process have been found to be significant [76]. The next important production process is provided by the Drell-Yan type charged Higgs pair production in

$$
p p \rightarrow q \bar{q} \rightarrow H^{+} H^{-},
$$


mediated by s-channel $\gamma$ and $Z$ boson exchange. The NLO QCD corrections which can be inferred from the Drell-Yan process are of moderate size. The SUSY-QCD corrections are mediated by virtual gluino and squark exchange in the initial state and are small [45]. Another source of charged Higgs pair production is the loopmediated process from $g g$ initial states [77, 78]

$$
p p \rightarrow g g \rightarrow H^{+} H^{-},
$$

known at LO only. It is of similar size as the bottominitiated process [78]

$$
p p \rightarrow b \bar{b} \rightarrow H^{+} H^{-},
$$

which relies on the introduction of bottom quark parton densities. The NLO corrections have been computed [79], and the SUSY-QCD corrections are of significant size. The pure QCD and genuine SUSY-QCD corrections can be of opposite sign. Another production mechanism is in association with a $W$ boson [80, 81]

$$
p p \rightarrow g g \rightarrow H^{+} W^{-} \quad \text { and } \quad \text { c.c. }
$$

The process, which is known at $\mathrm{LO}$ only, is mediated by top-bottom loops and, if their masses are light enough, by stop-sbottom loops. The corresponding bottom-quark initiated process in the five flavour approximation is $[80$, 82]

$$
p p \rightarrow b \bar{b} \rightarrow H^{+} W^{-} \quad \text { and } \quad c . c .
$$

The QCD are known and are of moderate size [83].

\section{CONCLUSIONS}

In summary, the important Higgs boson production cross sections at the LHC are theoretically under good control. Most (SUSY) QCD corrections are known. The corrections are large in several cases, and the remaining theoretical uncertainties have decreased from LO $100 \%$ down to $\lesssim 15 \%$. Considerable progress has also been made in the calculation of the dominant background processes up to NLO. Finally, there are a lot of programs available including many of these corrections.

\section{ACKNOWLEDGMENTS}

I would like to thank the organizers of SUSY08 for their support and hospitality.

\section{REFERENCES}

1. See e.g. M. Spira, Fortsch. Phys. 46 (1998) 203; A. Djouadi, Phys. Rept. 457 (2008) 1 and Phys. Rept. 459 (2008) 1; M. Gomez-Bock et al., J. Phys. Conf. Ser. 18 (2005) 74; M. Gomez-Bock et al., arXiv:0712.2419 [hep-ph].

2. P.W. Higgs, Phys. Lett. 12 (1964) 132 and Phys. Rev. 145 (1966) 1156; F. Englert and R. Brout, Phys. Rev. Lett. 13 (1964) 321; G.S. Guralnik, C.R. Hagen and T.W. Kibble, Phys. Rev. Lett. 13 (1964) 585.

3. Lower/Exp: [LEP Higgs WG], Phys. Lett. $B 565$ (2003) 61; higher/Th. e.g.: T. Hambye and K. Riesselmann, Phys. Rev. D55 (1997) 7255.

4. [LEP Higgs WG], arXiv:hep-ex/0107031 and Eur. Phys. J. C47 (2006) 547.

5. See e.g. G. Degrassi et al., Eur. Phys. J. C28 (2003) 133.

6. D. Graudenz, M. Spira and P.M. Zerwas, Phys. Rev. Lett. 70 (1993) 1372; M. Spira et al., Phys. Lett. B318 (1993) 347 and Nucl. Phys. B453 (1995) 17.

7. A. Djouadi, M. Spira and P.M. Zerwas, Phys. Lett. B264 (1991) 440; S. Dawson, Nucl. Phys. $B 359$ (1991) 283; S. Dawson and R. Kauffman, Phys. Rev. D49 (1994) 2298.

8. M. Krämer, E. Laenen and M. Spira, Nucl. Phys. B511 (1998) 523.

9. R.V. Harlander and W.B. Kilgore, Phys. Rev. Lett. 88 (2002) 201801 and JHEP 10 (2002) 017; C. Anastasiou and K. Melnikov, Nucl. Phys. B646 (2002) 220; V. Ravindran, J. Smith, W.L. van Neerven, Nucl. Phys. B665 (2003) 325.

10. S.Marzani et al., Nucl. Phys. B800 (2008) 127 and arXiv:0809.4934 [hep-ph].

11. S. Catani et al., JHEP 07 (2003) 028.

12. S. Moch and A. Vogt, Phys. Lett. B631 (2005) 48; V. Ravindran, Nucl. Phys. B746 (2006) 58 and Nucl. Phys. B752 (2006) 173.

13. A. Djouadi and P. Gambino, Phys. Rev. Lett. 73 (1994) 2528; U. Aglietti et al., Phys. Lett. B595 (2004) 432; G. Degrassi and F. Maltoni, Phys. Lett. B600 (2004) 255; S.Actis et al., arXiv:0809.1301 [hep-ph].

14. S. Dawson, A. Djouadi and M. Spira, Phys. Rev. Lett. 77 (1996) 16.

15. R.V. Harlander and M. Steinhauser, Phys. Lett. B574 (2003) 258, Phys. Rev. D68 (2003) 111701, JHEP 09 (2004) 066; R.V. Harlander and F. Hofmann, JHEP 03 (2006) 050.

16. M. Mühlleitner and M. Spira, Nucl. Phys. B790 (2008) 1.

17. C. Anastasiou et al., JHEP 01 (2007) 082; U. Aglietti et al., JHEP 01 (2007) 021.

18. C. Anastasiou, S. Beerli and A. Daleo, Phys. Rev. Lett. 100 (2008) 241806.

19. C.R. Schmidt, Phys. Lett. B413 (1997) 391; D. de Florian, M. Grazzini and Z. Kunszt, Phys. Rev. Lett. 82 (1999) 5209; V. Ravindran, J. Smith, W.L. Van Neerven, Nucl. Phys. B634 (2002) 247; C.J. Glosser and C.R. Schmidt, JHEP 0212 (2002) 016.

20. I. Hinchliffe and S. Novaes, Phys. Rev. D38 (1988) 3475; R.P. Kauffman, Phys. Rev. D44 (1991) 1415 and Phys. Rev. D45 (1992) 1512; C. Balazs and C.P. Yuan, Phys. Lett. B478 (2000) 192; E.L. Berger and J.W. Qiu, Phys. Rev. D67 (2003) 034026; A. Kulesza, G. Sterman,W. Vogelsang, Phys. Rev. D69 (2004) 014012; G. Bozzi et al., Phys. Lett. B564 (2003) 65, Nucl. Phys. B737 (2006) 73, Nucl. Phys. B791 (2008) 1. 
21. J.M. Campbell, R.K. Ellis and G. Zanderighi, JHEP 10 (2006) 028.

22. O. Brein and W. Hollik, Phys. Rev. D68 (2003) 095006; B. Field, S. Dawson and J. Smith, Phys. Rev. D69 (2004) 074013.

23. U. Langenegger et al., JHEP 0606 (2006) 035.

24. S. Catani, D. de Florian and M. Grazzini, JHEP 0201 (2002) 015.

25. C. Anastasiou, K. Melnikov and F. Petriello, Nucl. Phys. B724 (2005) 197.

26. C. Anastasiou, G. Dissertori and F. Stöckli, JHEP 0709 (2007) 018.

27. S. Catani and M. Grazzini, Phys. Rev. Lett. 98 (2007) 222002; M. Grazzini, JHEP 0802 (2008) 043; S Catani and M. Grazzini, arXiv:0802.1410 [hep-ph].

28. T. Binoth et al., Eur. Phys. J. C16 (2000) 311; C. Balazs et al., Phys. Rev. D76 (2007) 013009.

29. Z. Bern, L.J. Dixon and C. Schmidt, Phys. Rev. D66 (2002) 074018

30. M. Dittmar and H.K. Dreiner, Phys. Rev. D55 (1997) 167.

31. L.J. Dixon, Z. Kunszt, A. Signer, Phys. Rev. D60 (1999) 114037; J.M. Campbell and R.K. Ellis, Phys. Rev. D60 (1999) 113006.

32. M. Grazzini, JHEP 0601 (2006) 095.

33. S. Frixione and B.R. Webber, JHEP 0206 (2002) 029; S. Frixione et al., JHEP 0308 (2003) 007.

34. T. Binoth et al., JHEP 0503 (2005) 065; M. Dührssen et al., JHEP 0505 (2005) 064.

35. S. Dittmaier, S. Kallweit and P. Uwer, Phys. Rev. Lett. 100 (2008) 062003; J.M. Campbell, R.K. Ellis and G. Zanderighi, JHEP 0712 (2007) 056.

36. P. Nason et al., Nucl. Phys. B303 (1988) 607, Nucl. Phys. B327 (1989) 49, (E)-ibid. B335 (1990) 260; W. Beenakker et al., Phys. Rev. D40 (1989) 54; W. Beenakker et al., Nucl. Phys. B351 (1991) 507

37. W. Bernreuther et al. Phys. Rev. Lett. 87 (2001) 242002.

38. N. Kauer and D. Zeppenfeld, Phys. Rev. D65 (2002) 014021.

39. R. Frederix and M. Grazzini, Phys. Lett. B662 (2008) 353.

40. N.E. Adam et al., Higgs WG, arXiv:0803.1154 [hep-ph].

41. A. Bredenstein et al., Phys. Rev. D74 (2006) 013004 and JHEP 0702 (2007) 080.

42. R.N. Cahn and S. Dawson, Phys. Lett. B136 (1984) 196; K. Hikasa, Phys. Lett. B164 (1985) 341; G. Altarelli, B. Mele and F. Pitolli, Nucl. Phys. B287 (1987) 205.

43. T. Han, G. Valencia and S. Willenbrock, Phys. Rev. Lett. 69 (1992) 3274.

44. T.Figy, C. Oleari, D. Zeppenfeld, Phys. Rev. D68 (2003) 073005; T. Figy, D. Zeppenfeld, Phys. Lett. B591 (2004) 297; E.L. Berger, J. Campbell, Phys. Rev. D70 (2004) 073011; M. Ciccolini, A. Denner, S. Dittmaier, Phys. Rev. Lett. 99 (2007) 161803, Phys. Rev. D77 (2008) 013002.

45. A. Djouadi and M. Spira, Phys. Rev. D62 (2000) 014004.

46. W. Hollik et al., arXiv:0804.2676 [hep-ph].

47. V. Del Duca et al., Nucl. Phys. B616 (2001) 367.

48. V. Del Duca et al., JHEP 0610 (2006) 016.

49. T. Figy et al., JHEP 02 (2008) 076.

50. T. Plehn, D.L. Rainwater and D. Zeppenfeld, Phys. Lett. B454 (1999) 297 and Phys. Rev. D61 (2000) 093005.

51. J. Campbell and R.K. Ellis, Phys. Rev. D65 (2002) 113007; C. Oleari and D. Zeppenfeld, Phys. Rev. D69 (2004) 093004

52. N. Kauer et al., Phys. Lett. B503 (2001) 113.
53. B. Jäger, C. Oleari, D. Zeppenfeld, JHEP 07 (2006) 015.

54. S. Dittmaier, P. Uwer and S. Weinzierl, Phys. Rev. Lett. 98 (2007) 262002 and arXiv:0810.0452

55. Z. Kunszt, Nucl. Phys. B247 (1984) 339; J.F. Gunion, Phys. Lett. B261 (1991) 510; W.J. Marciano and F.E. Paige, Phys. Rev. Lett. 66 (1991) 2433.

56. W. Beenakker et al., Phys. Rev. Lett. 87 (2001) 201805 , Nucl. Phys. B653 (2003) 151; S. Dawson et al., Phys. Rev. D67 (2003) 071503.

57. W. Peng et al., arXiv:hep-ph/0505086

58. W. Hollik and M. Rauch, AIP Conf. Proc. 903 (2007) 117.

59. A. Bredenstein et al., JHEP 0808 (2008) 108.

60. ALTAS Coll., TDR, CERN/LHCC/99-15; CMS Coll., TDR, CERN-LHCC-2006-021.

61. S. Dittmaier, M. Krämer, M. Spira, Phys. Rev. D70 (2004) 074010; S. Dawson et al., Phys. Rev. D69 (2004) 074027, Phys. Rev. Lett. 94 (2005) 031802 and Mod. Phys. Lett. A21 (2006) 89.

62. D. Dicus et al., Phys. Rev. D59 (1999) 094016; C. Balazs, H.J. He and C.P. Yuan, Phys. Rev. D60 (1999) 114001.

63. R.V. Harlander and W.B. Kilgore, Phys. Rev. D68 (2003) 013001.

64. J. Campbell et al., Phys.Rev. D67 (2003) 095002.

65. J. Campbell et al., arXiv:hep-ph/0405302; C. Buttar et al., arXiv:hep-ph/0604120.

66. S Dittmaier et al., JHEP 0703 (2007) 114.

67. F. Boudjema and L.D. Ninh, Phys. Rev. D77 (2008) 033003.

68. G. Gao, R.J. Oakes and J.M. Yang, Phys. Rev. D71 (2005) 095005 .

69. S. Dawson, C.B. Jackson, Phys. Rev. D77 (2008) 015019.

70. S.L. Glashow, D.V. Nanopoulos and A. Yildiz, Phys. Rev. D18 (1978) 1724; Z. Kunszt, Z. Trocsanyi and W.J. Stirling, Phys. Lett. B271 (1991) 247.

71. T. Han and S. Willenbrock, Phys. Lett. B273 (1991) 167.

72. O. Brein, A. Djouadi and R. Harlander, Phys. Lett. B579 (2004) 149.

73. M.L. Ciccolini, S. Dittmaier and M. Krämer, Phys. Rev. D68 (2003) 073003.

74. A.C. Bawa, C.S. Kim, A.D. Martin, Z. Phys. C47 (1990) 75; F. Borzumati, J.-L. Kneur, N. Polonsky, Phys. Rev. D60 (1999) 115011; A. Belyaev et al., JHEP 0206 (2002) 059.

75. W. Peng et al., Phys. Rev. D73 (2006) 015012.

76. S.H. Zhu, Phys. Rev. D67 (2003) 075006; T. Plehn, Phys. Rev. D67 (2003) 014018; E.L. Berger et al., Phys. Rev. D71 (2005) 115012; G.P. Gao et al., Phys. Rev. D66 (2002) 015007.

77. S. Willenbrock, Phys. Rev. D35 (1987) 173; A. Krause et al., Nucl. Phys. B519 (1998) 85; Y. Jiang et al., J. Phys. G23 (1997) 385, (E) G23 (1997) 1151; O. Brein and W. Hollik, Eur. Phys. J. C13 (2000) 175.

78. A.A. Barrientos Bendezu and B.A. Kniehl, Nucl. Phys. B568 (2000) 305.

79. H. Hong-Sheng et al., Phys. Rev. D71 (2005) 075014.

80. A.A. Barrientos Bendezu and B.A. Kniehl, Phys. Rev. D59 (1999) 015009; O. Brein, W. Hollik and S. Kanemura, Phys. Rev. D63 (2001) 095001.

81. A.A. Barrientos Bendezu and B.A. Kniehl, Phys. Rev. D63 (2001) 015009.

82. D.A. Dicus et al., Phys. Rev. D40 (1989) 787.

83. W. Hollik and S.H. Zhu, Phys. Rev. D65 (2002) 075015; J. Zhao, C.S. Li and Q. Li, Phys. Rev. D72 (2005) 114008. 